\title{
Effects of geometry and temperature on mode I interlaminar fracture of filled polypropylene-elastomer nanocomposite
}

\author{
BISHNU P PANDA, SMITA MOHANTY and S K NAYAK* \\ Laboratory for Advanced Research in Polymeric Materials (LARPM), Central Institute of Plastics Engineering \\ and Technology, Bhubaneswar 751 024, India
}

MS received 18 July 2013; revised 8 October 2013

\begin{abstract}
In this study, organically modified Na-MMT clay was used for the preparation of blend nanocomposites containing different ratios of polypropylene (PP) and ethylene propylene diene monomer (EPDM) elastomer in a twin screw extruder. Maleic-grafted PP (MAPP) was used as compatibilizer for making PP hydrophilic. Surface modification of Na-MMT was made by using amino propyl trimethoxy silane (APS) and trimethyl amine as coupling agent with surface grafting catalyst, respectively. A fracture mechanics approach has been adopted by mode $I$ test and the effects of specimen geometry have been investigated. Increase in interlaminar fracture energy value, $G_{c}$, was observed as the crack propagated through the composite, i.e. a rising 'R-curve' for both blend and nanocomposites. Deep fracture studies were carried out at different temperatures $\left(-60^{\circ} \mathrm{C}\right.$ to $\left.60^{\circ} \mathrm{C}\right)$ using Izod impact and SENT tests. Fracture energy, fracture stress and brittle ductile transition were determined from crack initiation and propagation process, which showed significant improvement in impact and fracture energy at positive temperature. The wide-angle X-ray diffraction (XRD) patterns showed increased $d$-spacing of clay layers, indicating enhanced compatibility between PP and clay with the addition of maleated polypropylene (MAPP). Morphology/impact property relationships and an explanation of the toughening mechanisms were made by comparing the impact properties with scanning electron micrographs (SEMs) of fracture surfaces. The transmission electron microscopy (TEM) photomicrographs illustrated the intercalated and partially exfoliated structures of the hybrids with clay, MAPP and elastomer.
\end{abstract}

Keywords. Fracture toughness; brittle ductile transition; fractography; polypropylene.

\section{Introduction}

Polypropylene (PP) offers a very attractive combination of physical and mechanical properties at a relatively low cost, which makes it a versatile material with continuously increasing applications. However, not all the characteristics of this material are suitable for common service conditions. For instance, PP exhibits poor low temperature impact resistance, because of its high transition temperature and high crystallinity. In order to overcome these limitations, elastomers such as ethylenepropylene random copolymer (EPR) (Yazdani-Pedrem et al 2003), ethylene-propylene-diene terpolymer (EPDM) (López-Manchado et al 2001; Zhao and Dai 2002) poly(ethylene-co-1-octene) (Da Silva et al 2001), among others, have been added to PP as impact modifiers. The increase in toughness can be attributed to cavity formation in the rubbery particles when stress is applied, thus deforming the matrix through the formation of shear bands or crazes which can absorb energy (Bucknall and

*Author for correspondence (larpmcipet@gmail.com)
Paul 2000). Despite excellent toughening effects, the presence of rubbery particles decreases the modulus and yield strength and can increase the cost.

Further, due to incompatibility of PP with these elastomers, functionalized polymers have been used as blend compatibilizers to improve interfacial adhesion. Layered silicate clays used as fillers in polymers has gained considerable attention due to the ability to achieve exceptional property enhancements at very low-level loading. In recent years, a concerted effort has been made to disperse layered silicates in low-cost polyolefins, such as polypropylene (PP) (Alexandre and Dubois 2000; Reichert et al 2000). Many conventional fillers, e.g. talc and calcium carbonate, are used to enhanced various properties (Collar et al 2003). Due to size effect, large loading of such fillers significantly improved stiffness, which results in poor processibility. Using layer silicates in the polymers as a filler alleviated these issues and good level of dispersion was achieved with little modification. Layered silicate clays such as montmorillonite (MMT) have attracted much attention because of their unique properties, such as large aspect ratio, high surface area and low cost. Addition of low-level loading of silicate clays to 
polymers markedly improves their tensile strength, stiffness and heat resistance, facilitates processing and reduces component weight. However, in order to attain a qualitatively fine dispersion of the nanofiller within the matrix, a surface treatment of the filler should be considered (Rong et al 2004) or a polymeric compatibilizer should be added during melt mixing (Ben-Dor et al 2009). It has been found that the addition of nanometresized sodium montmorillonite clay (Na-MMT) significantly improves both the toughness and stiffness of PP, while having little effect on the tensile strength (Mehta et al 2004). The general idea behind this study is to mimic the rubber toughening mechanism using rigid filler particles. The rigid particles must debond and create free volume in the blend on a sub-micron size level. This is much like the cavitation mechanism in rubber toughened systems. Kim et al (2007) reported that the size of elastomer particles and the aspect ratio of the clay particles tended to decrease with increasing clay content. Additions of clay particles will make TPO matrix to stiffen and toughen. Organoclay additions are also considered to be beneficial for the enhancement of the mechanical properties of rubber-rich TPO blends. Nanocomposites containing $30 \mathrm{wt} \%$ elastomer undergo a brittle-ductile transition with increase in the amount of clay from 1 to $3 \mathrm{wt} \%$ and behave as super tough $(600 \mathrm{~J} / \mathrm{m})$ compared to the one containing $1 \mathrm{wt} \%$ marked. This apparent change is attributed to the reduction in elastomer size below the critical value that induces brittle-ductile transition (Jiang et al 2004). Mishra et al (2005) prepared TPO/clay nanocomposites in which the TPO contained $25 \mathrm{wt} \% \mathrm{PP}$ and 75 wt\% EPDM. They reported that the nanocomposites exhibited remarkable improvements in the tensile and storage moduli over their pristine TPO blend. Tjong and Bao (2007) showed a two-fold increase of impact fracture toughness of the HDPE/2\% Org-MMT and HDPE/4\% Org-MMT nanocomposites by adding 10\% SEBS-g-MA. Panda et al (2012) reported significant improvement in fracture stress and fracture energy by incorporation of $3 \mathrm{wt} \% \mathrm{TiO}_{2}$ clay in PP/EPDM (70/30) blend.

It is to ascertain the mechanism whereby nanoparticles lead to enhanced toughness. For a polymer matrix with rigid inorganic particles, an improvement in modulus is expected according to conventional rules of mixing. On the other hand, toughening by micron-sized inorganic particles can generally be explained by a crack deflection theory (Faber and Evans 1983). However, the application of these theories to nanometre-sized particles does not predict a toughness enhancement as the nanoparticles may be too small to effectively interact with the propagating crack (Cotterell et al 2007).

Toughness of polymers is often measured using Charpy and Izod impact test. However, the results of these tests depend upon specimen geometry. Although results are expressed as energy absorbed per unit area or energy absorbed per specimen width, they are known to vary non-proportionally with these dimensions. A method for the determination of fracture toughness has been developed by Marshall et al (1973) by calculating strain energy release rate, $G_{\mathrm{c}}$-directly from the results of impact tests on multiple specimens with different notch depths. Plati and Williams (1975) reported that critical strain energy release rate, $G_{\mathrm{c}}$, can be easily deduced from the absorbed energy measurement with assumption of elastic deformation from impact test. Results of impact test involve measurement of energy $(U)$ to break notched specimen, generally divided by ligament area $(A)$ to provide surface energy. In order to minimize the number of experiments, it is useful to evaluate toughness at least under two extreme conditions of temperature, i.e. under impact conditions and fracture toughness by SENT test methods. However, PP tested using the notched Izod method has a brittle-ductile transition below room temperature. This is similar to the glass transition temperature. The rate of clay loading also has a great effect on the brittle-ductile transition (van der Sanden et al 1994). Jiang et al (2004) reported that the brittle-ductile transition for the blends of PP, nylon 66 and HDPE with rubber is dependent on the interparticle distance, temperature, testing speed and matrix properties.

In this study, we seek to ascertain the deformation mechanisms associated with the increase in toughness and stiffness as a result of the inclusion of inorganic nanoparticles in a elastomer-modified PP matrix. Consideration was also made of the effect of these deformation processes upon strength. To promote exfoliation of clay platelets, a polypropylene-grafted-maleic anhydride (MAPP) material is premixed with the organoclay prior to melt processing. The effect of nanoclay on mode I type fracture toughness is studied and the characteristics of failure is examined by studying the structural changes taking place in the nanocomposites by SEM, TEM and XRD techniques. Furthermore, the fracture toughness behaviour of these nanocomposites is compared.

\section{Experimental}

\subsection{Materials}

Polypropylene copolymer, Repol B120MA of density 0.96 and melt flow index $12 \mathrm{~g} / 10 \mathrm{~min}\left(230{ }^{\circ} \mathrm{C} / 2.16 \mathrm{~kg}\right.$, ASTM D 1238), was supplied by Reliance Petrochemicals. Ethylene-propylene-diene rubber (EPDM) in pellets, medium molecular weight with ethylidene norbornene as termonomer (4.5\%), ethylene content of about 68\%, medium cure rate, was supplied by Dupont Dow elastomers under the trade name Nordel IP 4770. Na-MMT with cationic exchange capacity (CEC) of 92 mequiv/100 g was purchased from Southern Clay Products Inc., USA. Aminopropyltriethoxysilane $N$-(2-aminoethyl)-3-aminopropyl trimethoxy-silane (98\%, 


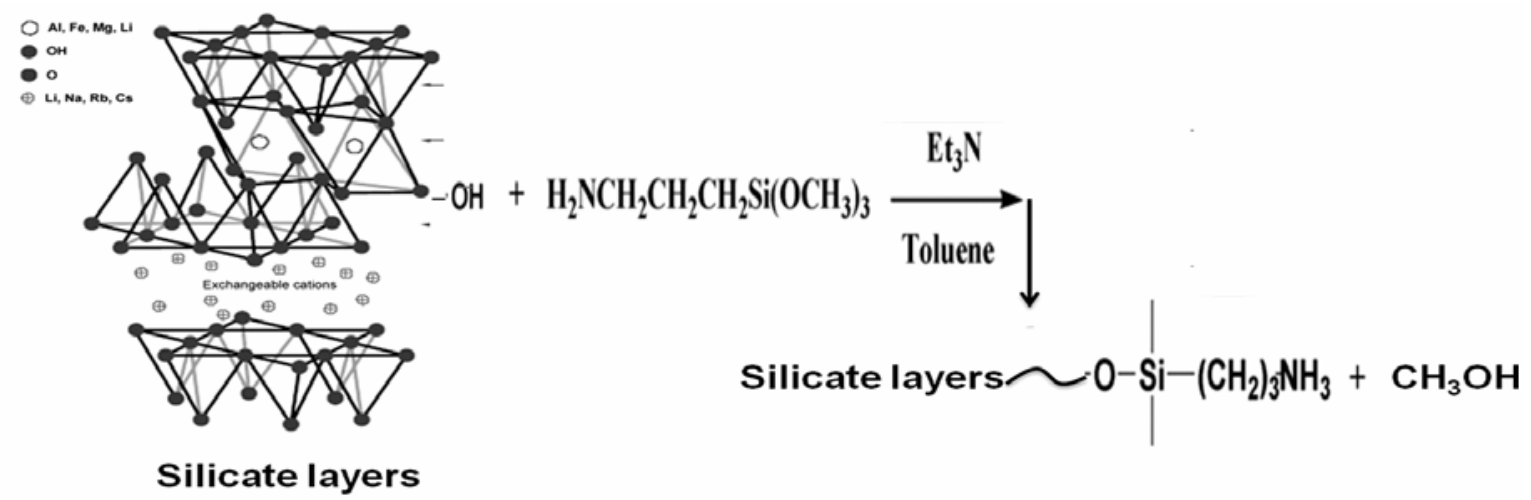

Figure 1. Treatment scheme of Na-MMT clay.

Merck) and triethyl amine ( $\mathrm{Et}_{3} \mathrm{~N}, 99 \%$, Merck) were used as coupling agent and surface grafting catalyst, respectively. Methoxy propyl acetate (MPA, 96\%), butyl acetate (99\%) and toluene (98\%) were obtained from Aldrich Chemicals.

\subsection{Surface modification of Na-MMT nanoparticle}

A total of $12 \mathrm{~g}$ of $\mathrm{Na}-\mathrm{MMT}$ nanoparticles were kept in a vacuum chamber for $24 \mathrm{~h}$ at $100{ }^{\circ} \mathrm{C}$ and then dispersed in $500 \mathrm{~mL}$ anhydrous toluene with the aid of ultrasonification for $30 \mathrm{~min}$. Triethyl amine of quantity $1.5 \mathrm{~mL}$ used as a catalyst and $15 \mathrm{wt} \%(1.5 \mathrm{~mL})$ aminopropyltrimethoxysilane (APS) were added, respectively, with constant stirring at $300 \mathrm{rpm}$ for $1 \mathrm{~h}$. The mixture was then refluxed for $5 \mathrm{~h}$ under nitrogen atmosphere. Modified particles were filtered under suction and physically adsorbed. APS compounds on the modified surface of nanoparticles were washed with ethanol for 4-5 times and then dried in a low-pressure oven at $80{ }^{\circ} \mathrm{C}$ for $12 \mathrm{~h}$. Reaction mechanism of the clay treatment has been depicted in figure 1 .

\subsection{Preparation of blend and its nanocomposites}

Different blends and nanocomposites were formed containing melt compounding mixture of PP, EPDM and a master batch material containing equal parts of maleated PP (MA-g-PP, contents 2-5 wt\%) and an organically modified MMT clay. Compounding was carried out using microcompounder (Xplore, DSM, The Netherlands) at screw speed of $60 \mathrm{rpm}$ and maintained constant torque level of $80 \mathrm{Nm}$ in all the cases. The resulting temperature during the processing of material was maintained at $190{ }^{\circ} \mathrm{C}$, followed by annealing in a vacuum oven at $80^{\circ} \mathrm{C}$ for $4 \mathrm{~h}$ duration. The first series of PP with EPDM blend was obtained at levels varying from 10 to $50 \mathrm{wt} \%$ in PP matrix. Extruded nanocomposite pellets were dried and then formed into standard tensile specimens (ASTM D638, type I) and impact specimens by Izod test (ASTM
D256) bars in an Explore injection moulding machine using barrel temperature $190^{\circ} \mathrm{C}$, injection pressure $40 \mathrm{bar}$ and holding pressure of 30 bar.

\section{$2.4 \quad F T-I R$ test}

FT-IR spectra of modified clay nanoparticles were recorded using Nicolet 6700, USA (FT-IR) spectrometer. Each spectrum was obtained by adding 64 consecutive scans with a resolution of $4 \mathrm{~cm}^{-1}$ within the range of 500 $4000 \mathrm{~cm}^{-1}$. Prior to the FT-IR analysis, samples were dried at $100{ }^{\circ} \mathrm{C}$ overnight. This powder mixture is then pressed in a mechanical press to form a translucent pellet through which the beam of the spectrometer can pass.

\subsection{Impact test}

An instrumented Izod type impact testing apparatus, CEAST, Italy, was used to study the impact strength of all the samples and the total energy observed by measuring potential energy lost as per ASTMD 256 standard. The hammer had a $3.65-\mathrm{kg}$ mass and the impact velocity was $1.92 \mathrm{~m} / \mathrm{s}$. Impact specimens of size $76 \times 12.7 \times 3.2 \mathrm{~mm}$ were machined and they were subsequently notched using a notch cutter to produce notch lengths of 1.5, 2.5, 3.5 and $4.5 \mathrm{~mm}$, respectively. The specimens were tested at different temperatures, ranging from $-60{ }^{\circ} \mathrm{C}$ to $+60{ }^{\circ} \mathrm{C}$. The brittle-to-ductile transition temperature was determined looking at the impact values as well as the fracture surface of the fractured specimens. For each kind of composition, five specimens were tested and the average value is reported.

\subsection{Fracture toughness by impact test method}

The total energy absorbed by the specimen related to fracture energy of the material expressed in terms of 'critical strain energy release rate' $\left(G_{c}\right)$ for situation like 
size of yield zone ahead of the crack tip is expressed by the following equation

$$
G_{\mathrm{c}}=\frac{U}{B D \phi},
$$

where $B$ is the specimen thickness; $D$ the specimen width; $\phi=C / d c / d(a / w)$ is a calibration factor which depends on the length of the notch and the size of the sample; $C$ the specimen compliance; $a$ the notch length; $G_{\mathrm{c}}$ value obtained from the slope of plot made by $U$ vs $B D \phi$ by series of the specimens with different notch length.

\subsection{Single-edge notch tensile test}

SEN tensile tests have been performed by using an Instron 3382 machine of rectangular bar specimens having dimension $74 \times 10 \times 4 \mathrm{~mm}$ according to ISO $180 / 1$. A single-edge $\mathrm{V}$-shaped notch of $2 \mathrm{~mm}$ depth and tip radius $0.25 \mathrm{~mm}$ was milled in the specimen after moulding. The specimen length between clamps was $45 \mathrm{~mm}$. Force, time and displacement data were recorded by piezo force transducer located between the crosshead and upper arm. Force-displacement signal results energy determination by calculating area under the curve. The tensile machine was equipped with environmental chamber to maintain temperature in the range of $-100-100{ }^{\circ} \mathrm{C}$. All measurements were performed in five-fold. Notched specimen was fractured at varying test temperature range between -60 and $+60{ }^{\circ} \mathrm{C}$.

The entire fracture process was divided into two stages. The crack initiation stage started from zero displacement and was assumed to take place up to the point of maximum stress. All displacements after this point of maximum force contribute to crack propagation, which is the second stage of the fracture process. Brittle fracture is characterized by zero propagation displacement.

Maximum stress: $\quad$ Maximum force on the

force-displacement curve
divided by the initial
cross-sectional area behind
the notch.

Crack initiation: Displacement of clamp between maximum force to the initial force.

Crack propagation: Displacement of clamp between the points of maximum force and zero force.

Fracture displacement: Summation of initiation and propagation displacements.

Fracture energy:

Summation of initiation and propagation energies.

\subsection{Thermo gravimetric analysis (TGA)}

Thermo gravimetric analysis (TGA) was used to investigate thermal decomposition behaviour of samples with a
SDT Q600 thermo gravimetric analyser (TA Instrument Inc., USA). Tests were done under nitrogen at a scan rate of $10^{\circ} \mathrm{C} / \mathrm{min}$ in a programmed temperature range of 30 $600{ }^{\circ} \mathrm{C}$. A sample of 5-10 mg was used for each run. The weight change was recorded as a function of temperature.

\section{$2.9 \quad X$-ray diffraction $(X R D)$}

The WAXD patterns were obtained using Shimadzu MAXima_XRD-7000 X-ray diffractometer $(40 \mathrm{kV}, 30 \mathrm{~mA}$ equipped with a $\mathrm{CuK} \alpha$ radiation source $(\lambda=1.54 \AA)$. The diffraction pattern of nanoclays as well as the blend nanocomposites were examined over a range of diffraction angle $2 \theta$ from 2 to $10^{\circ}$ with a step size of $0.02^{\circ}$. The peak positions were employed to obtain periodicities according to Bragg's law, $d=\lambda / 2 \sin \theta$, where $\lambda$ is the $\mathrm{X}$-ray wavelength $(\lambda \operatorname{CuK} \alpha=0.154 \mathrm{~nm})$ and $2 \theta$ is the scattering angle.

\subsection{Scanning electron microscopy (SEM)}

The SEM of impact fractured composite specimen was examined by using ZEISS EVO 60, Germany, at an acceleration voltage of $30 \mathrm{kV}$. To study the fracture topology under SEM, the fractured surface was coated with gold without etching or staining using a vacuum sputter coater prior to testing to improve the surface conductivity and to reduce charge build up on the surface.

\subsection{Transmission electron microscopy (TEM)}

The morphology of nanocomposite was investigated using a Philips CM300 transmission microscope (TEM: FEI Company, USA) at an accelerating voltage of $200 \mathrm{kV}$. Thin sections with thickness of $80 \mathrm{~nm}$ were cut from the fractured specimen by using a Leica EM UC7 ultramicrotome (equipped with a diamond knife under cryogenic condition) for subsequent TEM observations.

\section{Results and discussion}

\subsection{FT-IR analysis}

To confirm the nature of modification and functional group attachment, FT-IR analysis was carried out and the peaks were analysed as shown in figure 2. As observed from the spectrum, peak at $1196 \mathrm{~cm}^{-1}$ indicated the position characteristics of $\mathrm{Si}-\mathrm{O}-\mathrm{Si}$ stretching frequency. In general, since the coupling agent molecules are bound to the clay mineral, the strength of $\mathrm{Si}-\mathrm{O}$ vibration absorption should be increased. However, $\mathrm{Si}-\mathrm{O}$ vibration absorption in figure 2 is strong with a broad band centred at $1196 \mathrm{~cm}^{-1}$. Sharp band observed at $1035 \mathrm{~cm}^{-1}$ corresponds to symmetric and asymmetric bending of $\mathrm{Si}-\mathrm{O}-\mathrm{Si}$ 
(Yin et al 2010). Presence of peak at $1542 \mathrm{~cm}^{-1}$ further confirms the presence of amino group from the APTS on the modified nanoclay surface. By using extraction in ethanol solution, residual of APS has been removed and the mentioned peaks showed the grafting of the APS on the nanoparticles successfully.

\subsection{Notched Izod tests}

Figure 3 depicts influence of MMT clay and elastomer content on notched Izod impact strength of PP matrix. The presence of $40 \%$ elastomer reasonably increased the impact strength up to $175 \%$ compared to neat PP. This resulted in phenomenal transition from brittle to ductile behaviour of PP due to the presence of smaller elastomeric domains, leading to more toughness (Wahit et al 2006). However, decline in impact strength was observed beyond $40 \%$ EPDM content. This may be a result of poor dispersion and phase separation within the PP matrix, which contributes to a decrease in the impact energy. For nanocomposites containing up to $40 \mathrm{wt} \%$ elastomer, the addition of clay leads to an increase in the Izod values reaching a maximum at $3 \mathrm{wt} \%$, as shown in figure 3(a). This may be due to the ability of the filler to support stresses transferred from matrix. Decrease in impact strength is expected with increasing filler content; however, the opposite trend observed for the nanocomposites can be explained in terms of morphological changes induced by the dispersion of clay particles. However, at 5\% clay, there is little decline in the impact strength observed. The increase in impact strength may be ascribed to reduction in the size of elastomer particle due to barrier effect of clay.

At similar clay loading, PP/EPDM blend nanocomposites with MAPP have higher impact strength than similar composites without MAPP (figure 3b). This can be attributed to the better dispersion and adhesion of clay and PP/EPDM matrix. MAPP not only improves the miscibility

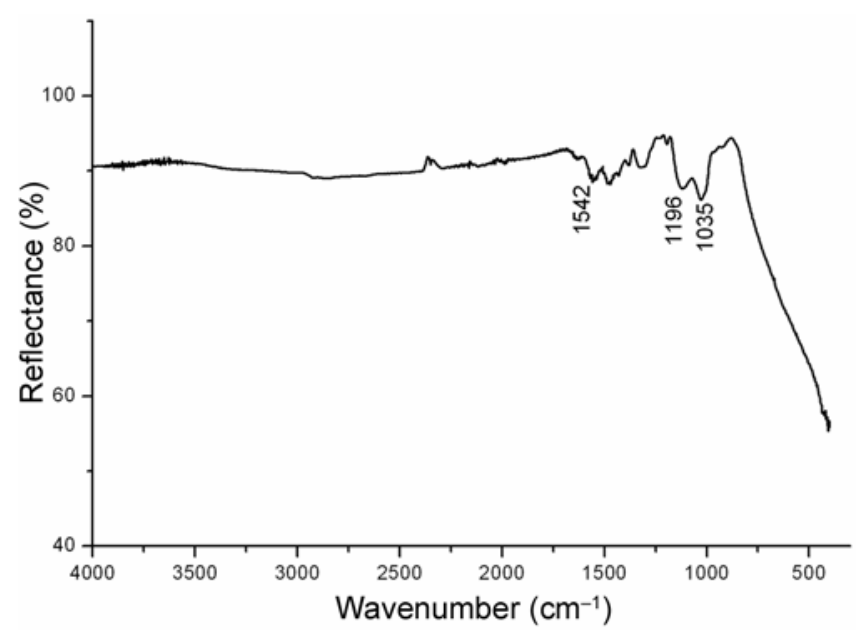

Figure 2. FT-IR spectra of the treated clay. between organically modified clay and PP, but also aids in expanding the clay galleries and enabling insertion of polymer chains, leading to formation of intercalated nanocomposite structure. The optimum impact property with addition of MAPP probably due to the fact that the modified clay is polar in nature with two hydroxyl groups in its structure, which interacts more easily with carboxyl group of MAPP. Hence, it is assumed that, within the blend matrix, nanoclay is distributed predominantly in PP phase. Impact strength of blend matrix increases up to $660 \mathrm{MPa}$, which indicates toughening effect of nanoclay layers within the blend matrix. Incorporation of MAPP further reduces the interfacial energy of both phases and provides a reactive site for intercalation of clay. The differences in chemical structure and organic modifier used in the nanoclay played a vital role in dispersion of layered silicates within the blend matrix.

Figure 4 shows a typical plot of total energy $U$ vs ligament area $(B D \phi)$ for each initial crack size of the PP/EPDM blend as well as PP/EPDM blend nanocomposites. Slope of the least-squares fitted line results value of the critical strain energy $\left(G_{c}\right)$. The result implies linear relationship between $G_{\mathrm{c}}$ for both binary blends and ternary composites. It is shown that the addition of clay to the PP/EPDM/MAPP blends exhibited higher $G_{\mathrm{c}}$ values than that of PP and PP/EPDM blends, which implies incorporation of clay-enhanced toughness of the binary blends (Gam et al 2004). Increased toughness was more prominently observed at $3 \mathrm{wt} \%$ of clay loading. This may be the result of better enhancement of compatibilization and adhesion effect of nanoclay due to the presence of MAPP in PP/EPDM matrix (Long and Shanks 1996). The compatibilizer and elastomer in the nanocomposite undergo cavitation, which releases triaxility at the notch and permits deformation by shear yielding. It is worth to note that the addition of clay as filler is expected to render high embrittlement to PP matrix. This implied synergistic toughening effect by the addition of clay into the PP/EPDM matrix with formation of encapsulation may be due to better interfacial interaction and unique molecular micro strength.

Fracture toughness value, $G_{c}$, of PP polymer continuously increased significantly from $1.3 \mathrm{~kJ} / \mathrm{m}^{2}$ for $\mathrm{PP}$ to $2.6 \mathrm{~kJ} / \mathrm{m}^{2}$ and $2 \cdot 1 \mathrm{~kJ} / \mathrm{m}^{2}$, respectively, for PP/EPDM/MAPP nanocomposite at $3 \mathrm{wt} \%$ and $5 \mathrm{wt} \%$ clay loading. This further indicated that the presence of MAPP as compatibilizer with clay-induced impact toughness on the elastomer-modified PP. Maximum improvement of $G_{\mathrm{c}}$ was 2 and 1.61 times, respectively, was observed in $3 \mathrm{wt} \%$ and $5 \mathrm{wt} \%$ nanoclay-filled PP composite, when compared with the virgin PP polymer. Even though the addition of nanoclay continuously increased the $G_{\mathrm{c}}$ values, the rate of increase was slower at higher clay concentration. The exfoliated structures contributed more toughness properties towards the loading directions due to the increase of the net aspect ratio. The increased value of $G_{\mathrm{c}}$ suggested 

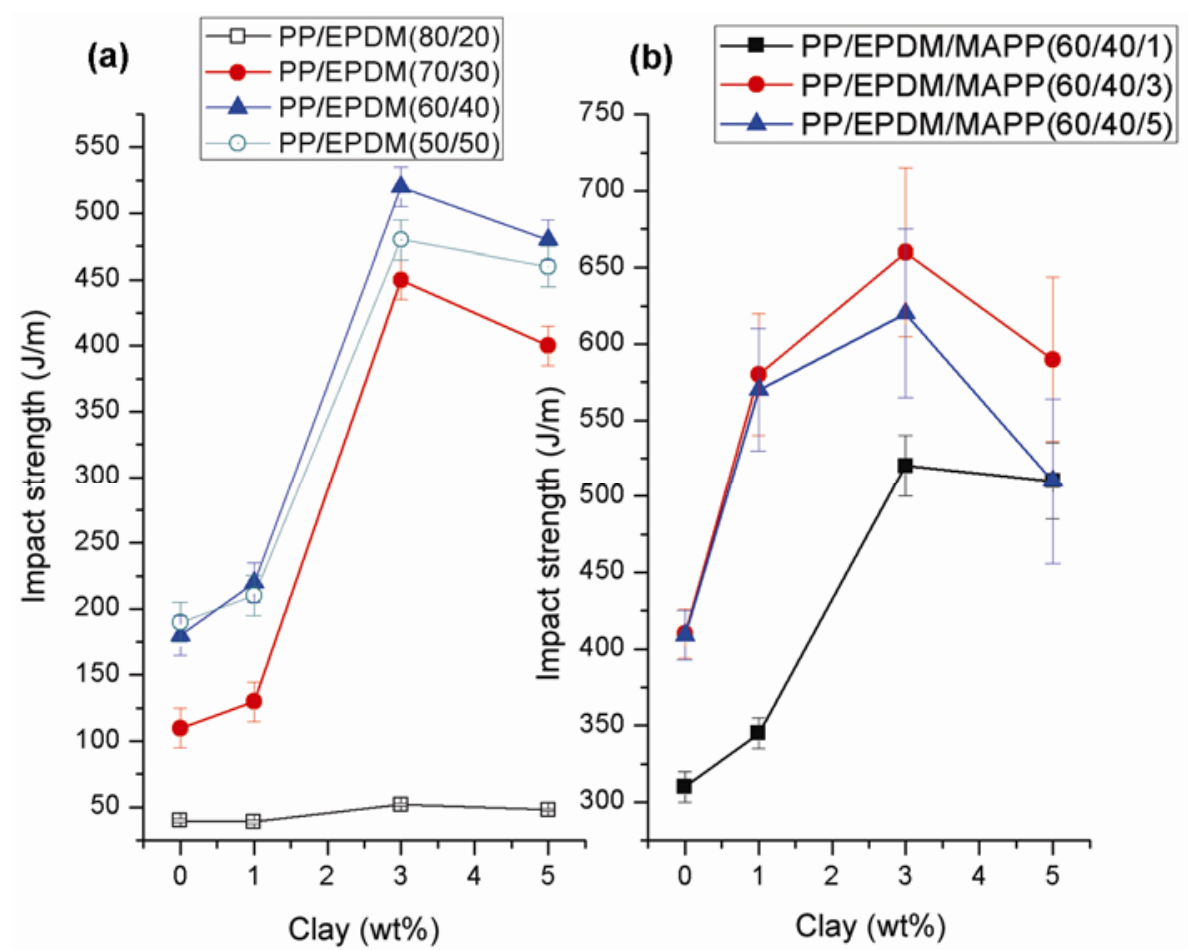

Figure 3. Effect of clay and elastomer content on notched impact strength of PP/EPDM blend nanocomposites containing variable content of (a) elastomer and (b) MAPP compatibilizer.

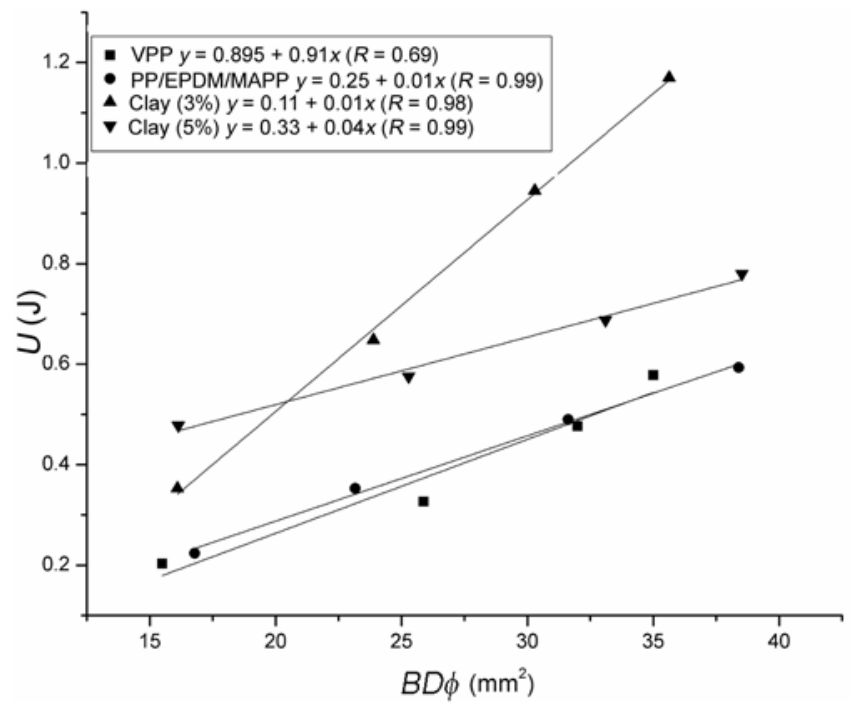

Figure 4. Plots of total energy vs 'corrected' ligament area for Izod impact test.

better stress transfer across the interfaces of blend containing rubber. However, a decrease in $G_{\mathrm{c}}$ value has been observed with further increase in nanoclay. It might be due to nanoclay particles interacting with each other and forming aggregations. Formation of aggregates causes premature cracks due to poor adhesion between nanoparticles and matrix, which reduced the toughness of the nanocomposites. From this, it can be concluded that the
$G_{\mathrm{c}}$ value for nanocomposite is very large as compared to VPP, depicting that large amount of energy dissipated during fracture per unit of newly created fracture surface area, which results in more fracture toughness of the blend nanocomposites. As depicted in figure 4, all slopes of the nanocomposites and blend provided strong $R$ curve behaviour $(R>0 \cdot 9)$. Both $3 \mathrm{wt} \%$ and $5 \mathrm{wt} \%$ clay loadings result in sharp slope with higher $G_{\mathrm{c}}$ value, which indicated more substantial resistance to crack growth.

Notched Izod impact tests were carried out in five-fold with all PP/EPDM blends and blend nanocomposites at different temperatures ranging from -60 to $60{ }^{\circ} \mathrm{C}$. The brittle to ductile transition temperature was determined by referring the impact values and fracture surface at different temperatures. Figure 5 illustrates the impact strength at various temperatures for PP/EPDM blends and $\mathrm{PP} /$ $\mathrm{EPDM} / \mathrm{Cl} 30 \mathrm{~B}$ nanocomposites. At low temperature, PP virgin and PP/EPDM (60/40) blend showed lower Izod impact values. However, impact values increased gradually with increasing temperature.

The impact value of $6.7 \mathrm{~kJ} / \mathrm{m}^{2}$ was observed for $40 \%$ EPDM loading at $-60{ }^{\circ} \mathrm{C}$. PP/EPDM (60/40) blend undergo the transition as the temperature decreases in the range between -40 and $0{ }^{\circ} \mathrm{C}$ close to the glass transition temperature of $\mathrm{PP}$. The intermediate temperature $(-40$ to $60{ }^{\circ} \mathrm{C}$ ) did not show distinct difference in fracture energy. Further, inclusion of MAPP compatibilizer to the PP/EPDM (60/40) blend enhanced absorbed energy during notched high-speed fracturing. At a high temperature 
$\left(40^{\circ} \mathrm{C}\right.$ ), the PP/EPDM/MAPP blend showed tough fracture of about $15 \mathrm{~kJ} / \mathrm{m}^{2}$ impact value. At low temperature $\left(-20^{\circ} \mathrm{C}\right)$ samples failed in mix mode with two of the samples exhibiting hinge break and failure in ductile mode with high energy absorbed. The samples that fail in ductile mode have average energy value three times that of samples, which fall in brittle mode.

Uniform PP/EPDM blend nanocomposites were studied, incorporating clay at different ratios of 1,3 and $5 \mathrm{wt} \%$ respectively, in notched Izod tests as a function of test temperature. The Izod impact values were low at low temperature with clay contents. With increasing content of nanoparticles, impact values increased, high at $40^{\circ} \mathrm{C}$ temperature observed. Blend nanocomposite containing $3 \mathrm{wt} \%$ of clay performed tough fracture and higher impact value of about $18 \mathrm{~kJ} / \mathrm{m}^{2}$. The PP polymer showed very low Izod value of $3.4 \mathrm{~kJ} / \mathrm{m}^{2}$ at $60{ }^{\circ} \mathrm{C}$. The intermediate temperature $\left(-60-40^{\circ} \mathrm{C}\right)$ for all compositions showed more distinct differences between the blends, whereas PP had constant low-level impact values. The PP/EPDM/ MAPP blends containing $1 \mathrm{wt} \%$ and $5 \mathrm{wt} \%$ of clay did not show this unusual maximum Izod values.

\subsection{Brittle/ductile transition}

The brittle to ductile transition temperature $\left(T_{\mathrm{bd}}\right)$ is defined as the onset of ductile deformation. At low temperature $\left(-40{ }^{\circ} \mathrm{C}\right)$, more EPDM elastomer content up to $40 \mathrm{wt} \%$ with MAPP compatibilizer improved the Izod properties. However, presence of enough elastomer relieves triaxial stress state in the material and prevents elimination. At $-40{ }^{\circ} \mathrm{C}$, plane stress condition of PP/EPDM/MAPP blend is changed to plane strain condition that limits contribution of additional rubber on the Izod values at this temperature. The transition temperature increased with increasing clay content in the PP/EPDM blend. At 3 wt\%

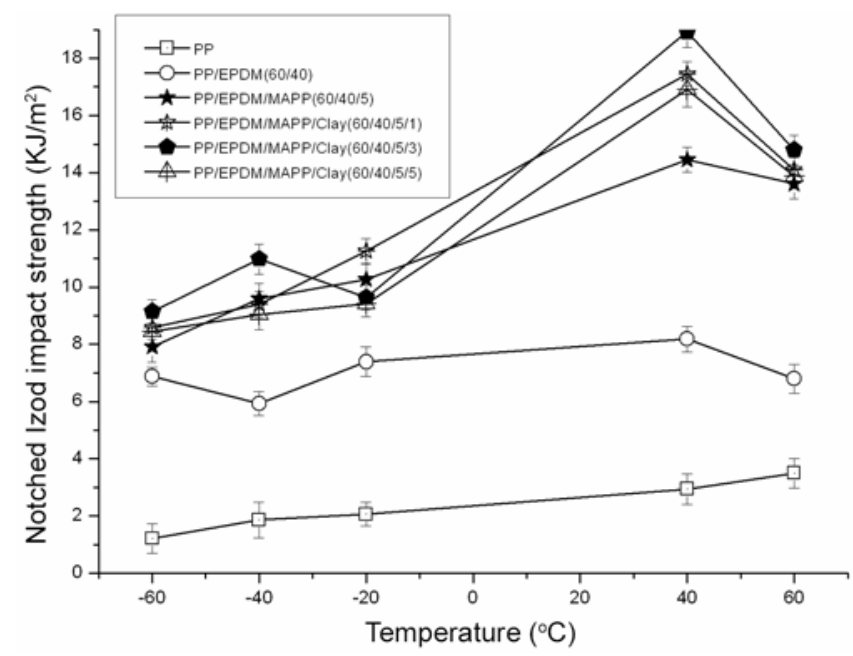

Figure 5. Notched Izod impact values at different temperatures. of clay concentration, this increase was strongest. Therefore, the addition of more than $3 \mathrm{wt} \%$ of clay to the PP/ EPDM/MAPP blend makes no contribution to the notched Izod values. The impact values as a function of temperature appeared to go through a maximum at higher temperature changes from brittle to ductile as well as a delamination on the addition of clay nanoparticle.

The impact values as a function of temperature appeared to go through a maximum at higher temperature between -20 and $60{ }^{\circ} \mathrm{C}$. At -60 to $-20{ }^{\circ} \mathrm{C}$, the blend nanocomposite shows the brittle region and at temperature between -20 and $60{ }^{\circ} \mathrm{C}$ ductile region is observed. It is expected that the increase in the amount of filler result in a decrease in impact strength of a composite material; however, the trend appeared to be opposite for this nanocomposites. Increase in impact strength is observed with increasing clay content. This unexpected change can be explained in terms of morphological changes induced by the dispersion of clay particles in the matrix. The apparent increase in impact strength of nanocomposite containing higher amount of elastomer at $3 \mathrm{wt} \%$ clay concentration most likely results from the reduction in the size of the elastomer particles, which may enhance the 'barrier' effect exerted by the clay particles preventing coalescence of elastomer particles during the melt processing (Chow et al 2003).

\subsection{Single-edge notch tensile tests}

SENT tests were performed at different temperatures for different wt\% of clay loading in the PP/EPDM/MAPP blend as depicted in figure 6. VPP without rubber content has a very low fracture stress. Adding $3 \mathrm{wt} \%$ of clay content in the PP/EPDM blend increased the fracture stress significantly and also changed from brittle to ductile fracture. The SENT graph shows $13 \mathrm{MPa}$ maximum stress for VPP increased up to $17 \mathrm{MPa}$ at $40^{\circ} \mathrm{C}$ by adding $40 \mathrm{wt} \%$ of EPDM elastomer. From the graph, the stress of 17.8 MPa was observed with addition of MAPP compatibilizer, which is 6\% greater than PP/EPDM (60/40) blend. Further, addition of clay increased the strength of the material. Little variation in the maximum stress observed in the ductile region remained between -20 and $40{ }^{\circ} \mathrm{C}$ temperature, due to the delamination. The stress completely decreased for $5 \mathrm{wt} \%$ clay loading up to $60{ }^{\circ} \mathrm{C}$. Maximum stress value of $3 \mathrm{wt} \%$ clay reinforced $\mathrm{PP} / \mathrm{EPDM} / \mathrm{MAPP}$ blend is $21 \mathrm{MPa}$ at $40^{\circ} \mathrm{C}$, further decreased up to $20 \mathrm{MPa}$ at $60^{\circ} \mathrm{C}$. At low temperature, maximum stress was about $16 \mathrm{MPa}$, increased up to $20 \mathrm{MPa}$ at high temperature, due to adhesion effect of clay.

Figure 7 illustrates comparison between fracture energy of SENT test and Izod impact test at different temperatures. Based on propagation results, the brittleductile transition temperature in SENT was determined as 
occurring between -20 and $40{ }^{\circ} \mathrm{C}$ for the PP/EPDM/MAPP blend nanocomposite containing $3 \mathrm{wt} \%$ clay. This also corresponds to $T_{\mathrm{bd}}$ of $40^{\circ} \mathrm{C}$ which was found in notched Izod testing. At low temperature, initiation energy increased slightly with temperature, but decreased with increasing temperature in the ductile range. At higher temperatures, yield stress decreases, leading to lower energy absorption. The difference between the fracture energy of SENT and impact tests possibly originates from the different way of loading the material in Izod and SENT tests. In SENT, material is loaded purely in the tensile direction, while in Izod, bending action is performed (William 1987). SENT and Izod tests comparison depends on the temperature. SENT test results in the fracture energy value about $15 \mathrm{~kJ} / \mathrm{m}^{2}$ at $-40^{\circ} \mathrm{C}$ and then

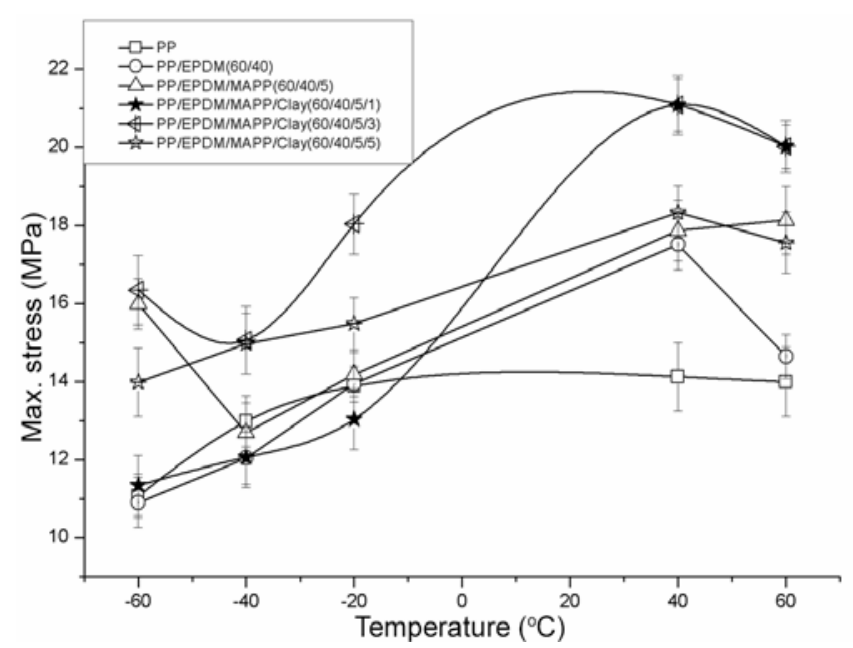

Figure 6. Influence of temperature on SENT results of blends and nanocomposites.

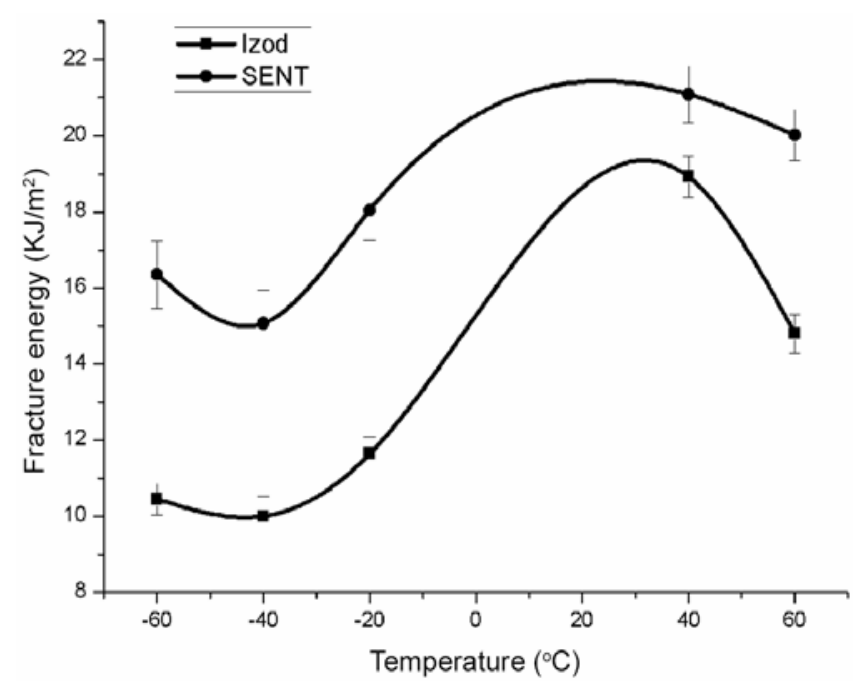

Figure 7. SENT test fracture energy results $(10 \mathrm{~m} / \mathrm{s})$ compared to notched Izod results for PP/EPDM (60/40) blend containing $3 \mathrm{wt} \%$ clay. suddenly increases up to $21 \mathrm{~kJ} / \mathrm{m}^{2}$ at $40{ }^{\circ} \mathrm{C}$. Similarly, for the Izod impact test, the fracture energy was $10 \mathrm{~kJ} / \mathrm{m}^{2}$ at $-40{ }^{\circ} \mathrm{C}$ and increased up to $18.9 \mathrm{~kJ} / \mathrm{m}^{2}$ at $40^{\circ} \mathrm{C}$. On comparison, SENT test showed the highest value at $40^{\circ} \mathrm{C}$, which is the optimized temperature.

\subsection{Fracture by SENT tests}

SENT tests were conducted at $10 \mathrm{~m} / \mathrm{s}$ and at room temperature for blends containing different $w \mathrm{t} \%$ of clays. Without rubber, PP shows a very low fracture stress. The fracture displacement for initiation and propagation increases sharply with loading of clay nanoparticles. The optimum value is $7 \mathrm{~mm}$ for propagation, $10 \mathrm{~mm}$ for initiation and $17 \mathrm{~mm}$ for total fracture was observed by inclusion of $3 \mathrm{wt} \%$ of clay nanoparticle in the PP/EPDM/ MAPP blend system. With increasing clay content, fracture displacement increased steadily (figure 8(a)). Transition from brittle to ductile behaviour took place between 1 and $3 \mathrm{wt} \%$ of clay reinforcement. Increased fracture displacement was observed at $3 \mathrm{wt} \%$ clay reinforced nanocomposite due to extensive yielding at the crack-tip because of highly deformed and elongated PP/EPDM blend particles to several hundred percent. Propagation results clearly showed brittle-to-ductile transition behaviour of the nanocomposites. Addition of clay content to the blend system enhanced in both initiation and propagation displacements in the ductile region.

Fracture energy increased with clay content up to $3 \mathrm{wt} \%$ in the PP/EPDM/MAPP blend (figure 8b). Brittle fracture showed no delamination marks on the fracture surface. Increasing the rubber content resulted in less delamination in the ductile region. The optimum value is $28 \mathrm{~kJ} / \mathrm{m}^{2}$ for propagation, $53 \mathrm{~kJ} / \mathrm{m}^{2}$ for initiation, and $81 \mathrm{~kJ} / \mathrm{m}^{2}$ for its total fracture energy was observed for $3 \mathrm{wt} \%$ of clay reinforced PP/EPDM/MAPP blend. Increasing the clay content provided the delamination in the ductile region. It was due to the rubber stretch out in the plain strain situation such that fluctuation of delamination took place.

\subsection{Thermo gravimetric analysis (TGA)}

The evolution of the weight loss of PP, PP/EPDM/MAPP blend and blend nanocomposite containing $3 \mathrm{wt} \%$ clay has been studied by TGA as shown in figure 9 . The temperature at which the weight loss was $5 \%$ can be considered as the initial decomposition temperature $\left(T_{\mathrm{d}}\right)$. Table 1 summarizes the thermal analysis data such as temperature at $5 \%$ weight loss $\left(T_{5 \%}\right)$, decomposition temperature at maximum weight loss rate $\left(T_{\max }\right)$, weight loss determined at the end of decomposition $(R) . T_{\text {onset }}$ varies from $283^{\circ} \mathrm{C}$ for neat PP to $373{ }^{\circ} \mathrm{C}$ for compatibilized PP/EPDM blend and $390{ }^{\circ} \mathrm{C}$ for blend nanocomposite containing $3 \mathrm{wt} \%$ clay, respectively. All the samples exhibit a very sharp 

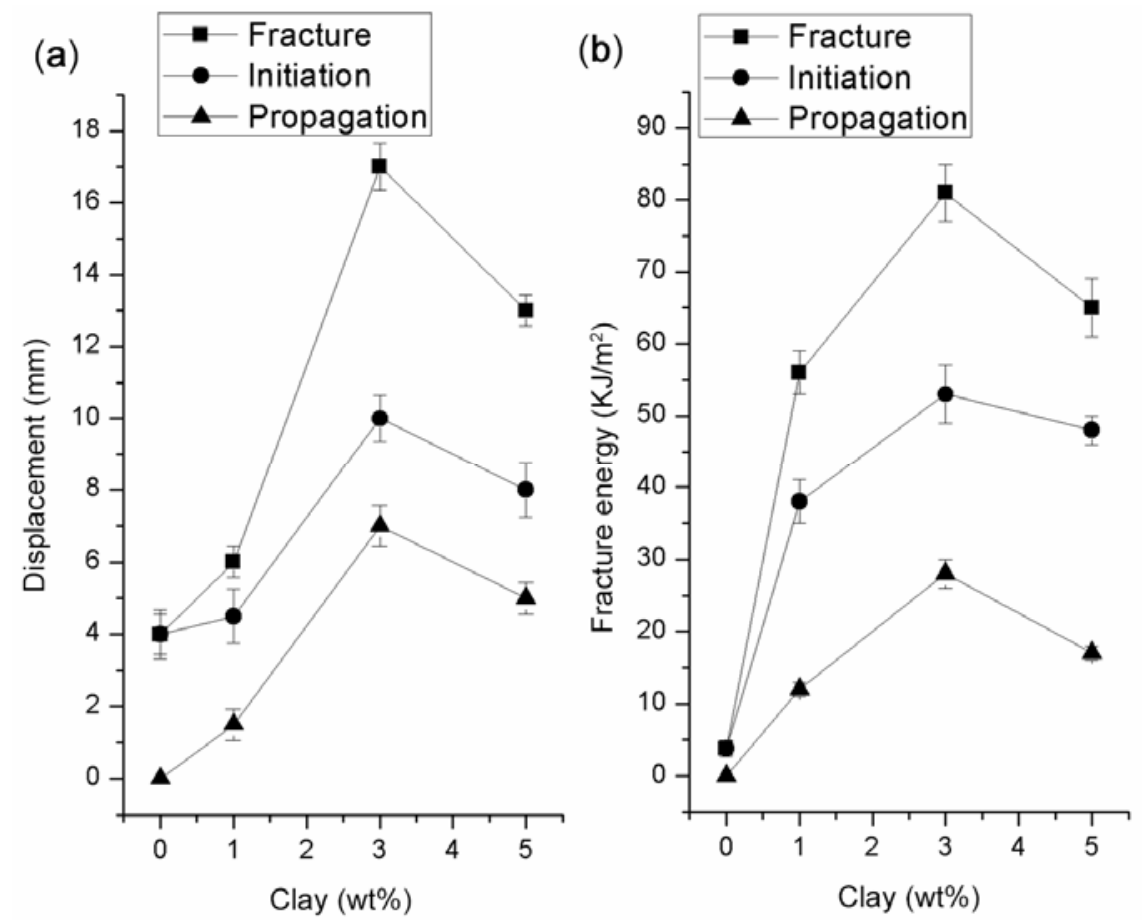

Figure 8. SENT results of PP/EPDM/MAPP blend nanocomposites: influence of clay contents on (a) displacement of crack initiation, propagation and fracture and (b) energy of crack initiation, propagation and fracture.

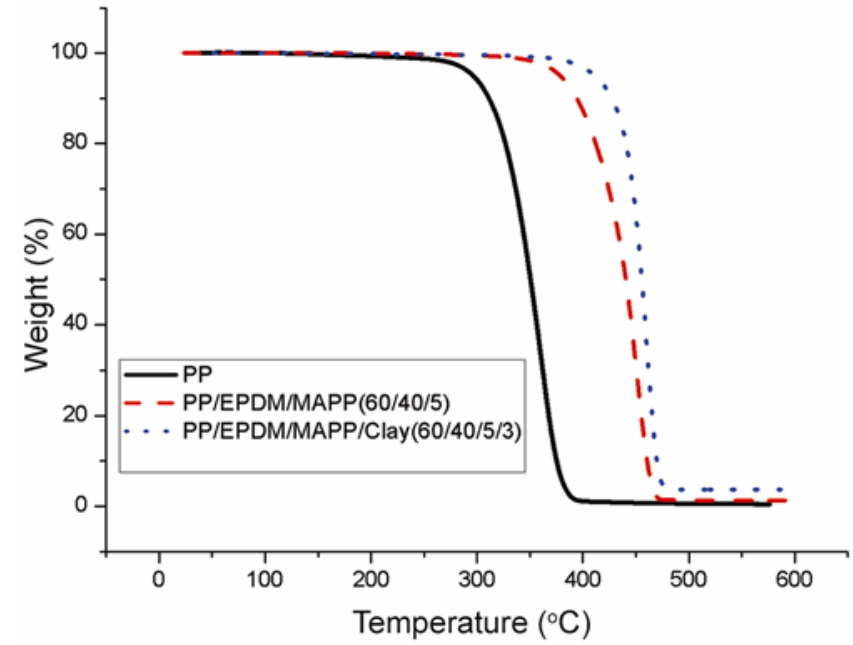

Figure 9. TGA curves of PP, PP/EPDM blend and blend nanocomposite containing $3 \mathrm{wt} \%$ clay.

Table 1. TGA characteristics of PP/EPDM blend and blend nanocomposite.

\begin{tabular}{lccc}
\hline Sample & $T_{5 \%}\left({ }^{\circ} \mathrm{C}\right)$ & $T_{\max }\left({ }^{\circ} \mathrm{C}\right)$ & $R(\%)$ \\
\hline PP & 333 & 391 & 0.45 \\
PP/EPDM/MAPP (60/40/5) & 378 & 468 & 1.3 \\
$\begin{array}{l}\text { PP/EPDM/MAPP/clay } \\
\quad \text { (60/40/5/3) }\end{array}$ & 411 & 477 & 3.61 \\
\hline
\end{tabular}

weight loss of about $95 \%$ between 330 and $470{ }^{\circ} \mathrm{C}$. As it can be noticed that the thermal stability of PP/EPDM (60/40) blend containing $3 \mathrm{wt} \%$ nanoclay and $5 \mathrm{wt} \%$ compatibilizer was increased slightly compared to initial sample PP. Also, higher char residue value up to $3.61 \mathrm{~g}$ was obtained for the nanocomposite sample. This small increase in thermal stability can be attributed to the clay nanolayers which can proceed as barriers to reduce the permeability of volatile degradation products from polymer matrix. From table 1, it can be seen that the nanoclay practically does not decompose during processing or characterization of these materials.

\subsection{WAXD results}

The XRD pattern of the blend nanocomposites at different clay concentration are shown in figure 10 . The prestine organoclay showed distinct diffraction peaks which correspond to interlayer spacing. The peaks of the blend nanocomposite with 1-3 wt\% clay has been shifted to a higher value (maximum $2 \theta=4 \cdot 1^{\circ}, d$-spacing $=$ $2 \cdot 2 \mathrm{~nm}$ ), which indicates an increase of $d$-spacing for clay layers. With increasing concentration of clay, compatibility between neat PP and clay may be enhanced and the polymer chains could be well diffused into the clay layers and $d$-spacing of clay layers might be increased. At higher clay contents, the viscosity increases even further and it can be assumed that the extra shear force generated 
by high viscosity will increase the basal spacing of clay platelets (Fornes et al 2001). Further, much wider peak observed for nanocomposite containing $3 \mathrm{wt} \%$ nanoclay confirms improvement in compatibility and the enhancement of intercalation from the better shear action results wider $d$-spacing. In general, the larger $d$-spacing at higher clay contents is attributed to the increased intercalation/exfoliation sites with increased clay content that might impede the higher basal spacing or exfoliation of individual silicate layers (Nam et al 2001). Intercalation and good dispersion of nanosize clay particles restrict the mobility of the polymer chains under loading, results in good interfacial adhesion that attributes the improvement in strength and modulus values as observed from mechanical results. On the other hand, the peaks of nanocomposite with $5 \mathrm{wt} \%$ nano clay were shifted to a lower value $\left(2 \theta=4.5^{\circ}, d\right.$-spacing $\left.=1.9 \mathrm{~nm}\right)$, resulting in a decrease in $d$-spacing for clay layers. This decrease of the $d$-spacing of clay layers is presumably due to low compatibility at interfaces between neat PP and clay. Agglomeration of clay layers at higher concentration may also contribute to reduce the $d$-spacing value. The decreasing rate of strength and elastic modulus with higher clay content can be attributed to the presence of unexfoliated aggregates.

\subsection{Fractography}

The investigation of surface morphology has been carried out by using scanning electron microscopy (SEM) to analyse the micro deformation and crack propagation mechanisms. Micrograph corresponding to the PP/EPDM/MAPP blend and the nanocomposite based on $3 \mathrm{wt} \%$ of clay are shown in figure 11 after stretching. Figure 11(a) shows partial miscibility of PP/EPDM/MAPP (60/40/3) blend and indicating that the seemingly detached elastomeric

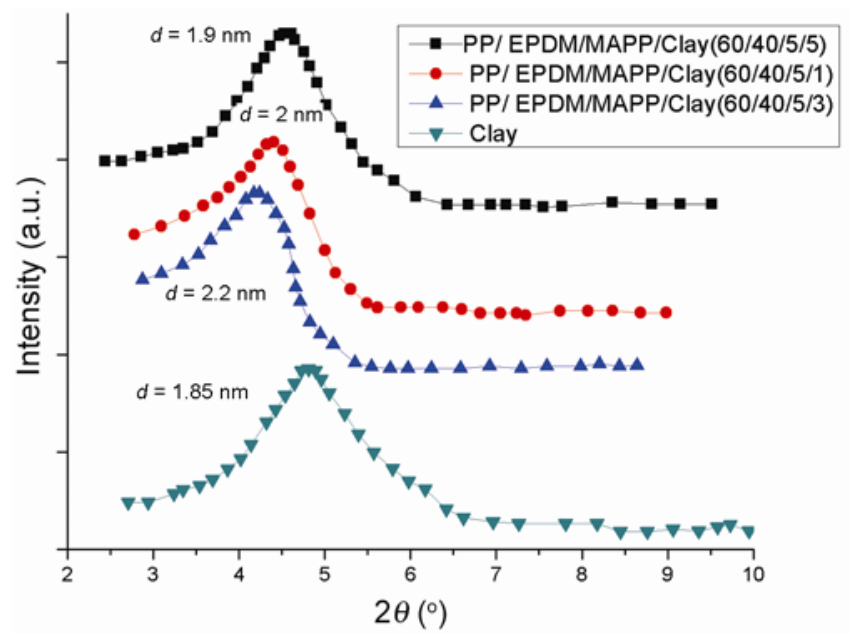

Figure 10. XRD patterns of nanocomposite containing different $w t \%$ of clay concentrations. particles are distinctly smaller than the holes of the PP host particles and much additional energy is required for propagating the crack. The presence of continuous phase containing a dispersed phase with nodules having spherical shapes of different sizes were observed (figure 11a). Presence of nodules exhibits a smooth aspect, while the continuous phase exhibits a rough surface. Some elongated voids are observed, which may probably be due to cavitations of rubber particles induced by the deboning. The higher value of impact strength observed may be due to the consequence of this elongated phase morphology. Presence of the EPDM elastomer results in considerable plastic deformation as expected. This effect depends on particle size of the droplets, dispersion, properties of the elastomer and their interaction. According to Liang and Li (2000), when rubber was grafted with a suitable content of maleic anhydride (MAH), the rubber particles were dispersed uniformly in the continuous PP phase with encapsulated thin layer. When highly dispersed, the rubber phase acts as an effective stress concentrator and enhances both crazing and shear yielding in the matrix.

Figure 11(b) depicted quite homogeneously dispersed nanofiller in the case of PP/EPDM blend nanocomposite, although some agglomerated are clearly recognizable. Wetting of the particles by the polymer matrix is clearly improved by the coupling treatment, making the interface between two phases almost distinguishable. The enhancement of the interfacial adhesion can be explained by a decrease in surface energy of the filler with silane coupling agent in the presence of MAPP compatibilizer, which leads to a better compatibility. The higher value of fracture toughness and impact strength obtained for the maleated PP may arise as a consequence of elongated or distorted outline of the holes in the matrix. The significant toughness may be ascribed due to the presence of polar functional group (maleic anhydride) which has improved the miscibility and adhesion between the EPDM and organoclay (Wahit et al 2006). In order to have efficient stress transfer between the two phases, the rubber particles should be well dispersed so that they can act efficiently as impact modifier, and they should also be well bonded to the polymer matrix ( $\mathrm{Li}$ et al 2001). Further presence of $3 \mathrm{wt} \%$ nanoclay does not show any brittle type failure mechanism, but rather shows extended and elongated deformations before failure. This deformation mechanism possibly induced more time for crack to failure. This result further suggests that there existed a structural change in the nanocomposite as clay content increased in the PP polymer matrix. This could have resulted in the improved fracture toughness properties. Also, the addition of nanoparticles in the blend results in much rougher surface, i.e. additional energy is required for fracture. It substantiates that crack deflection is responsible for the increase in fracture toughness, and an independent measure of the deflection process is necessary. Significantly dispersed rubber phase acts as an 

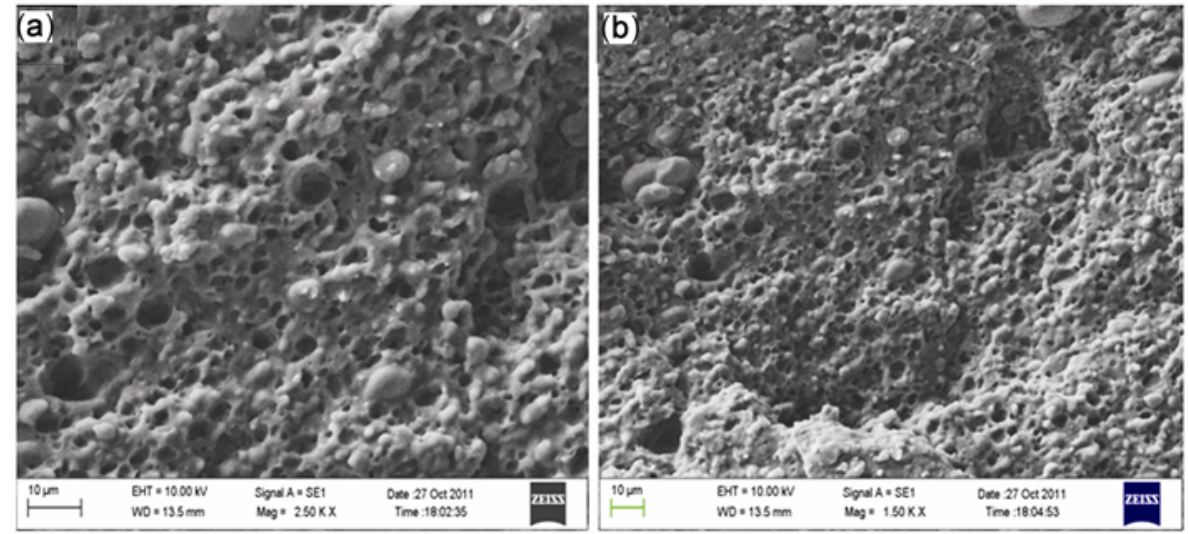

Figure 11. (a) SEM pictures of impact fracture surface of PP/EPDM/MAPP (60/40/5) blend and (b) PP/EPDM/MAPP/clay (60/40/5/3) blend nanocomposite at room temperature.
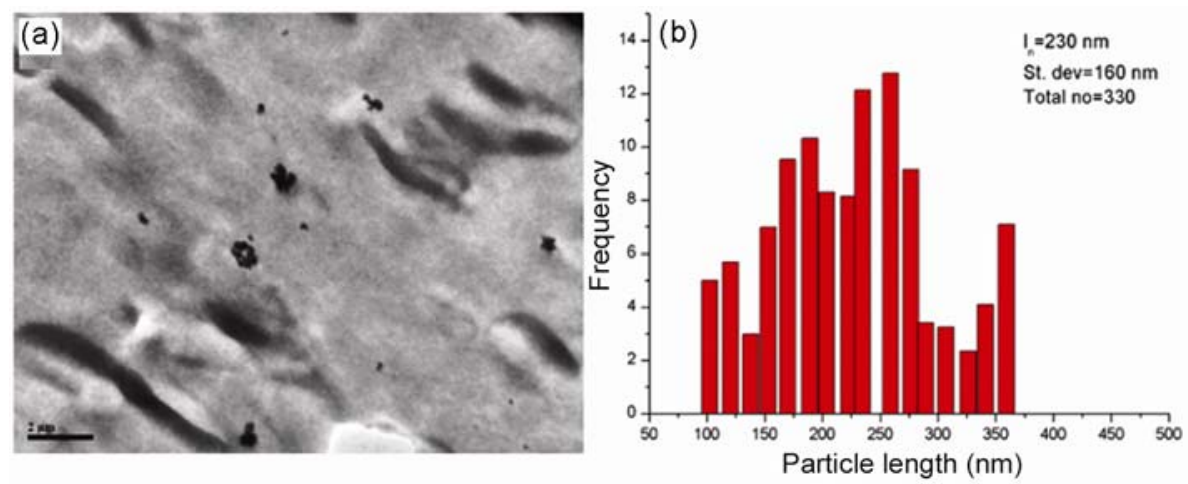

Figure 12. (a) TEM micrograph of PP/EPDM blend nanocomposite at 3 wt\% clay. (b) Histogram of MMT particle length obtained by analysing TEM micrograph.

effective stress concentrator and enhances both crazing and shear yielding in the matrix. The mechanism of energy dissipation was dominated by de-bonding, which coalesced to form transverse cracks.

Toughening behaviour of polymer strongly depends on characterization of elastomer particles, e.g. size, shape and distribution for the nanocomposite and blends. Recently, Mehta et al (2004) reported that the addition of organoclay caused a reduction in rubber particle size in $\mathrm{PP} /$ elastomer blends. They attributed reduction in rubber particle size with increment of melt viscosity as clay loading increases, acting as a chemical modifier for the rubber phase. In the developed TPO nanocomposite system, effect of elastomer size by the addition of clay has been carried out. The average particle size $\left(d_{\mathrm{w}}\right)$ of PP domains was calculated in the representative zones of the cryogenically fractured impact specimens from 200 particles by using following equation:

$$
d_{\mathrm{w}}=\frac{\sum n d^{2}}{\sum n d},
$$

where $n$ is the number of particles with size $d$. The weighed average particle size for PP/EPDM (60/40) blend was found to be $5.8 \mu \mathrm{m}$. Large interparticle distance may result in the localization of deformation. These large particles may contain several cavities situated near the interface with the matrix. As observed by Chou et al (1988), these large particles may contain more number of voids at the rubber matrix interface, which may reduce fracture energy. Further, interparticle distance for nanocomposite containing $3 \mathrm{wt} \%$ clay reduced to $1.27 \mu \mathrm{m}$, which reduced the interfacial tension and suggested good adhesion between clay and matrices (Jiang et al 1998). Lazzeri et al (1995) showed that the stabilization effect of composite will be stronger, when interparticle distance decreases as small particles undergo higher straining due to increase in the thickness of dilation band. Smaller particle size and stronger clay/rubber/matrix interaction results in high fracture in resistance. This favours the formation of diffused network of a large number of dilation bands corresponding to dissipated energy at whitened zone.

Figure 12(a) shows TEM micrographs of PP/EPDM/ MAPP blend nanocomposite containing $30 \mathrm{wt} \%$ elastomer and $3 \mathrm{wt} \%$ clay. Presence of clay leads to an apparent reduction in the size of the elastomer particles and results dramatically in a change in morphology. 
Much smaller elastomer particles appearing in the micrograph could be comparable to those clay platelets. Also, it is noted that a large portion of the nanoclay particles appeared as bent, skewed and/or curved structures with combination of intercalated stacks and exfoliated particles. The dark lines in figure 12(a) correspond to the intersection of clay layers $\sim 3.15 \mathrm{~nm}$ thick size containing a large number of multilayered clay platelets intercalated with PP as corroborated in the WAXD results. This result is closely related to the enhancement of clay dispersion by MAPP and the intercalation of clay platelets with polymer.

To evaluate clay platelet size, series of histograms of clay particle lengths and statistical data were obtained by viewing the section parallel to the transverse direction. Particle length of around 100-300 particles were taken for this analysis. As seen from these histograms, there exists a sizeable distribution for all frequency ranges. The clay platelet length ranging from 100 to $380 \mathrm{~nm}$ for different frequency ranges were observed. The average clay platelet length $\left(I_{\mathrm{n}}\right)$ appeared to be $230 \mathrm{~nm}$, which is a similar observation as reported by Nam et al (2001) for PP-gMA/clay nanocomposites system.

Figure 13 shows schematically a change in morphology of PP/EPDM blend nanocomposite as observed from SEM at different clay concentrations. Reduction in the size of the elastomer with incorporation of clay is believed to be responsible for enhancement of the properties of the nanocomposite. Nodules that appeared as spherical shapes of different sizes exhibit a smooth aspect induced
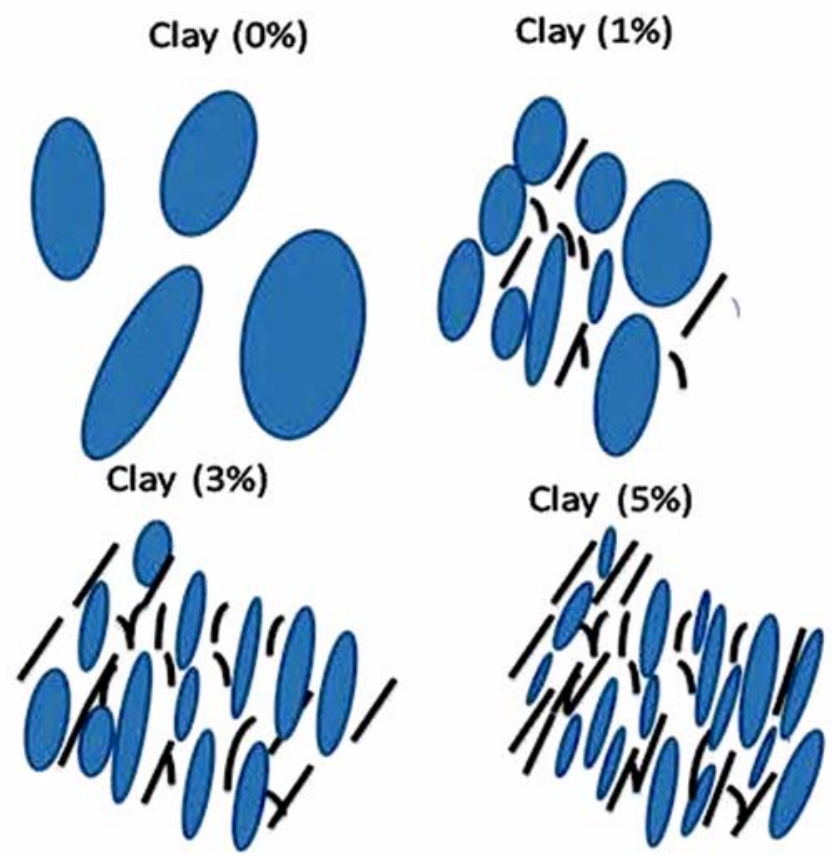

Figure 13. Schematic illustration of the morphological change for PP/EPDM blend nanocomposite with increasing clay content at a fixed elastomer concentration. with higher impact strength due to the consequence of elongated phase morphology. Highly dispersed rubber phase acts as an effective stress concentrator and enhances both crazing and shear yielding in the matrix. In addition to this, presence of clay particles retards the coalescence of small elastomer particles to form larger one, resulting in a reduction in the elastomer particle size. Kim et al (2007) demonstrated that dispersed clay particles caused reduction in particle size and stability of the dispersed elastomer domains in a nylon 6 matrix via formation of barrier to coalescence mechanism. Retardation of elastomer coalescence by clay particles is the main factor for the reduction of elastomer particle size.

\section{Conclusions}

PP polymer filled with EPDM elastomer and nanoclay at various concentrations (1, 3 and $5 \mathrm{wt} \%$ ) was prepared by using melt blending extrusion method. It was observed that up to $3 \mathrm{wt} \%$ of nanoclay in the PP matrix with presence of $30 \mathrm{wt} \%$ EPDM elastomer and $5 \mathrm{wt} \%$ MAPP compatibilizer reasonably increased the impact strength that resulted in a phenomenal transition from brittle to ductile behaviour of PP. The value of fracture energy, $G_{c}$, for all blends and nanocomposites generally increases as the crack propagated, i.e. a rising of ' $R$-curve' is observed. The increase of $G_{\mathrm{c}}$ reveals that there is a significant improvement in toughness, indicating that elastomer-modified PP materials with clay particle have toughening effect and nanocomposites are less brittle in nature. At the brittle-ductile transition, the fracture process changed from being a fast unstable with craze formation to a slow stable fracture showing ductile tearing. Significant changes in blend morphology and toughness were observed when MAPP was applied. The fractographic analysis confirmed a strong particle matrix adhesion and also revealed that nanoparticles did affect fracture behaviour of PP in various ways at different temperatures.

\section{References}

Alexandre M and Dubois P 2000 Mater. Sci. Eng. 281

Ben-Dor G, Dubinsky A and Elperin T 2009 Composites Part B: Eng. 40181

Bucknall C B and Paul D R 2000 Polym. Blends (John Wiley \& Sons) p. 243

Chou C J, Vijayan K, Kirby D, Hiltner A and Baer E $1988 \mathrm{~J}$. Mater. Sci. 1988232533

Chow W S, Mohd Ishak Z A, Karger-Kocsis J, Apostolov A A and Ishiaku U S 2003 Polymer 447427

Collar E P, Laguna O, Areso S and García-Martínez J M 2003 Europ. Pol. J. 39157

Cotterell B, Chia J Y H, Hbaieb K 2007 Eng. Fract. Mech. 74 1054

Da Silva A L N, Rocha M C G, Coutinho F M B, Bretas R E S and Scuracchio C 2001 J. Appl. Polym. Sci. 791634 
Faber K T and Evans A G 1983 Acta Metall. 31565

Fornes T D, Yoon P J, Keskkula H and Paul D R 2001 Polymer 429929

Gam K T, Miyamoto M, Nishimura R and Sue H J 2004 Polym. Eng. Sci. 431635

Jiang W, Liu C H, Wang Z G, An L J, Liang H J and Jiang B Z 1998 Polymer 393285

Jiang W, Yu D and Jiang B 2004 Polymer 456427

Jiang W, Yu D, An L and Jiang B 2004 J. Polym. Sci. Part B: Polym. Phys. 421433

Kim D H, Fasulo P D, Rodgers W R and Paul D R 2007 Polymer 485308

Lazzeri A and Bucknall C B 1995 Polymer 362895

Li F Q, Kim D G, Wu D Z, Lu K and Jin R G 2001 Polym. Eng. Sci. 412155

Liang J Z and Li R K Y 2000 J. Appl. Polym. Sci. 77409

Long Y and Shanks R A 1996 J. Appl. Polym. Sci 611877

López-Manchado M A, Kenny J, Quijada R and YazdaniPedram M 2001 Macromol. Chem. Phys 2021909

Marshall G P, Williams J G and Turner C E 1973 J. Mater. Sci. 8949

Mehta S, Mirabella F M, Rufener K and Bafna A 2004 J. Appl. Polym. Sci. 92928
Mishra J K, Hwang K J and Ha C S 2005 Polymer 461995

Nam P H, Maiti P, Okamoto M, Kotaka T, Hasegawa N and Usuki A 2001 Polymer 429633

Panda B P, Mohanty S and Nayak S K and Pandit S 2012 Int. J. Plast Tech. 1689

Plati E and Williams J G 1975 Polym. Eng. Sci. 15470

Reichert P, Nitz H, Klinke S, Brandsch R, Thomann R and Mülhaupt R 2000 Macromol. Mater. Eng. 2758

Rong M Z, Zhang M Q, Pan S, Lehmann B and Friedrich K 2004 Polym. Int. 53176

Tjong S C and Bao S P 2007 Compos. Sci. Tech. 67314

van der Sanden M C M, de Kok J M M and Meijer H E H 1994 Polymer 352995

Wahit M U, Hassan A, Mohd Ishak Z A, Rahmat A R and Abu Bakar A 2006 J. Thermoplast. Compos. Mater. 19545

Wahit M U, Hassan A, Rahmat A R, Lim J W and Mohd Ishak Z A 2006 J. Reinforce Plast. Compo. 25933

William J G 1987 Fracture mechanics in polymer (Wiley: New York)

Yazdani-Pedram M, Quijada R and López-Manchado M A 2003 Macromol. Mater. Eng. 288875

Yin H, Chen D and Chen D 2010 Polym. Eng. Sci. 50312

Zhao R and Dai G 2002 J. Appl. Polym. Sci. 862486 